\title{
Capillary wicking in bio-based reinforcements undergoing swelling - Dual scale consideration of porous medium
}

\author{
H.N. Vo ${ }^{\text {a }}$, M.F. Pucci ${ }^{\mathrm{b}}$, S. Corn ${ }^{\mathrm{b}}$, N. Le Moigne ${ }^{\mathrm{c}}$, W. Garat ${ }^{\mathrm{a}}$, S. Drapier ${ }^{\mathrm{a}}$, P.J. Liotier ${ }^{\mathrm{a}, *}$ \\ ${ }^{a}$ Mines Saint-Etienne, Université de Lyon, CNRS, UMR 5307 LGF, Centre SMS, 158 Cours Fauriel - 42023, Saint-Etienne, France \\ ${ }^{\mathrm{b}}$ LMGC, IMT Mines Ales, Université de Montpellier, CNRS, 6 Avenue de Clavières, 30319 Ales Cedex, France \\ ${ }^{\mathrm{c}}$ Polymers Composites and Hybrides (PCH), IMT Mines Ales, 6 Avenue de Clavières, 30319 Ales Cedex, France
}

\begin{abstract}
A B S T R A C T
Capillary wicking of liquids in natural fibrous reinforcements is a significant phenomenon in fibre-reinforced composites manufacturing through Liquid Composite Moulding (LCM) processes. Such phenomenon is however difficult to analyse due to heterogeneous, multiscale and variable fibrous medium during flow. Taking into account the fibre swelling, a modified Washburn's model is proposed to predict the capillary rise of liquids in flax fibres. Particularly, swelling and wicking are studied at two different scales, i.e. elementary fibres and individual yarns. Swelling effects have been considered in the model and a modified Washburn's theory has been developed. The proposed model satisfactory fits experimental results from capillary wicking tests, and hold for a $60 \%$ fibre volume fraction $\left(V_{f}\right)$ of fabric on a relatively short duration while it fits well on a longer duration for lower $V_{f}$.
\end{abstract}

Keywords:

A. Biocomposite

Natural fibre

E. Liquid composite moulding

Capillary wicking

\section{Introduction}

The use of natural fibres as reinforcements in composites has grown in the last decades. Indeed, natural fibres have interesting mechanical and damping properties, low density, and better sustainability [1-5]. In some cases, e.g. flax fibres, the specific modulus is comparable or even higher than for glass fibres [6]. The potential of flax fibres as reinforcements in structural composites can only be realized by taking advantage of twistless long fibre yarns. Besides, structural composite parts with high mechanical performances can only be obtained if high fibre volume fraction is reached. Therefore, Liquid Composite Moulding (LCM) processes are considered as promising and effective to manufacture structural composite parts reinforced with natural fibres.

In LCM processes, such as resin transfer moulding and resin infusion, the impregnation of fibrous reinforcements by resin is a crucial step contributing to the quality and performances of composite parts [7-9]. Those processes allow the manufacturing of large parts suitable for transportation industry applications $[10,11]$. The interest of composites reinforced by natural fibres in the transportation industry is due to their relatively high specific properties [12]. Fibrous reinforcements are commonly considered as porous media, and they can be ideally approximated as a capillary tube network $[13,14]$. Hence, wicking in those media can be described by the Washburn's theory [15], where the medium porosity is considered as constant, e.g. for synthetic fabrics. In the case of natural fibres fabrics, fibres can be subjected to swelling during wicking, especially with polar liquids, leading to changes in the porous medium configuration [16,17]. Natural fibres also have some limitations compared to synthetic fibres such as intrinsic hydrophilic character, low thermal stability, large variability in terms of morphology, chemical composition, and surface properties [18]. The absorption of liquids during LCM processes results in an increase of fibre diameter [16]. Subsequently, the pore size in between fibres decreases which changes the flow characteristics of resin during the impregnation process. Therefore, the swelling of natural fibres should take into account in the Washburn's equation to better describe the wicking phenomenon in these specific porous media. The behaviour of fibres in terms of swelling will be different in resin then in water. Even if dynamic wetting of cellulosic substrates by liquid polymer has been studied recently [19], it has been chosen to use water as test liquid because it represents the worst possible case for swelling of natural fibres.

Swelling of natural fibres has been studied extensively using different measurement techniques such as optical microscopy, automated laser scanning, or X-ray micro-computed tomography [16,20-24]. The effect of swelling on permeability of the fibrous medium has also been studied $[16,25]$. Several natural fibres, used as reinforcements in composites, have been studied such as flax, hemp, sisal, jute, and wood. Swelling measurements were performed on elementary fibres, fibres bundles or yarns (case of flax) immersed in water (in optical

\footnotetext{
* Corresponding author.

E-mail address: Liotier@emse.fr (P.J. Liotier).
} 
microscopy, automated laser scanning device) or placed in high relative humidity environment (in tomography machine). Optical microscopy and automated laser scanning directly give apparent diameter measurements while X-ray micro-computed tomography require image post analysis, but allow 3D reconstruction of fibre morphology. Particularly, with optical microscopy, fibre diameter was measured manually by picking two points at the edge of a single elementary fibre, and monitored over time during swelling. As a result, the fibre diameter as a function of time was obtained and the swelling rate could be estimated $[16,21]$.

Medium porosity is defined as spaces between fibres, that is to say all spaces that are not occupied by fibres. It can be calculated as the complement of a ratio of the volume of fibres on the overall volume $(\varepsilon$, the porosity, is $1-V_{f}$; $V_{f}$ being the fibre volume fraction). Depending on scales, such spaces can be considered as in between yarns, fibre bundles, and elementary fibres $[7,16,17,21,26]$. As pointed out above, the conventional Washburn's equation could not describe wicking for natural fibres absorbing water $[13,14,27]$, because the porous medium changes over time due to fibre swelling. More precisely, it was shown that the geometric product $(c \bar{r})$ in Washburn's equation related to tortuosity $c$ and mean porous radius $\bar{r}$ changed during wicking time. Therefore, the characteristic product $(c \bar{r})$ of the porous medium should take into account swelling effects and the Washburn's equation is modified accordingly.

In a previous work [21], a model accounting for the swelling of elementary fibres (related to the geometrical product) was proposed. However, both swelling of yarns and elementary fibres, and then spaces in between elementary fibres and individual yarns, should be considered to describe the porous structure of fibre network and its evolution during wicking [17]. Therefore, in the present work, a modified Washburn's model is proposed, taking into account the porous media and the swelling effects. Particularly, the swelling effects on wicking are evaluated at two scales, i.e. the scale of elementary fibres and the scale of individual yarns, based on optical microscopy and X-ray tomography measurements. Furthermore, the evolution of the spaces in between elementary fibres and individual yarns are also accounted in the model. Experimental wicking data were obtained with a tensiometer in the warp direction of quasi-unidirectional carbon and flax fabrics with $60 \%$ $V_{f}$. It should be noticed that $V_{f}$ of $60 \%$ was chosen in this study because structural composites with high mechanical performance are commonly processed at high $V_{f}$. In the experiment of wicking, such $V_{f}$ corresponds to the ratio of the volume of fibres to the internal volume of the cylindrical sample holder. Then, the swelling behaviour of flax fibres in water was characterized at both scales, i.e. elementary fibres and yarns, as input experimental data to establish a modified model of wicking adapted to natural fabrics and evaluate its validity at high fibre volume fraction.

\section{Theory}

\subsection{Conventional Washburn's equation for fabrics}

A liquid rise in porous media is usually described by Washburn's equation [15]. This equation models the complex porous structure of fibre network as bundles of capillary tubes in a cylindrical holder [13]. The model predicts the flow front position $h$ of liquid as a function of time:

$h^{2}(t)=\left[\frac{(c \bar{r})}{2}\right] \frac{\gamma_{L} \cos \theta_{a}}{\eta} t$

where $c$ is a parameter taking into account tortuosity, $\bar{r}$ is a mean porous radius, $\theta_{a}$ is an apparent advancing contact angle, $\gamma_{L}$ and $\eta$ are the liquid surface tension and viscosity, respectively. time:

Eq. (1) can be rewritten as a function of the square mass gain over

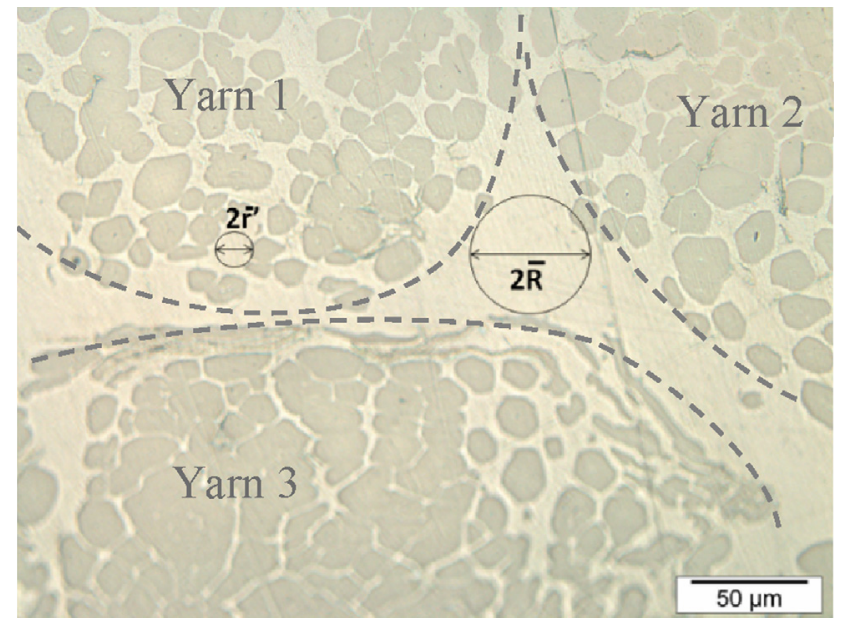

Fig. 1. Illustration of the two-scale pore radii in the cross-section of an epoxy based composite reinforced by quasi-unidirectional flax fabrics. Broken lines are added to visualize individual yarns.

$m^{2}(t)=\left[\frac{(c \bar{r}) \varepsilon^{2}\left(\pi R^{2}\right)^{2}}{2}\right] \frac{\rho^{2} \gamma_{L} \cos \theta_{a}}{\eta} t$

where $\varepsilon$, the porosity, is $1-V_{f}\left(V_{f}\right.$ : the fibre volume fraction), $R$ is the radius of a cylindrical sample holder, and $\rho$ is the liquid density. The squared-brackets term is related to both morphology of the porous medium and geometry of sample holder. Eq. (2) fits very well to the experimental data for synthetic fabrics because the medium porosity (related to geometric product $c \bar{r}$ ) remains constant over time [28].

\subsection{Model development}

For natural fibres, the porous medium changes over time during wicking of water due to fibre swelling. In a first approach, assuming that tortuosity is not affected by liquid and that the volume of lumen is neglected, hence the capillary wicking only takes place in the spaces between individual yarns and between elementary fibres. The dual scale capillary radius is then proposed as follows:

$c \bar{r}=c\left(\left(1-\varnothing_{\text {yarn }}\right) \bar{R}+\varnothing_{\text {yarn }} \bar{r}^{\prime}\right)$

where $\bar{R}$ and $\bar{r}^{\prime}$ are respectively the mean capillary radii between individual yarns, and between elementary fibres (Fig. 1), and $\varnothing_{\text {yarn }}$ is the surface ratio of yarns for a cross-section of the composite.

During wicking in natural porous media, the mean capillary radius was assumed to decrease linearly over time $t$ due to swelling $[13,14,21,27]$. In line with this approach, the swelling effects on the evolution of the mean capillary radii $\bar{R}$ and $\bar{r}^{\prime}$ should also be described. Eq. (3) can therefore be expressed as follow:

$(c \bar{r})(t)=c\left(\left(1-\varnothing_{\text {yarn }}\right)\left(\bar{R}_{0}-b t\right)+\varnothing_{\text {yarn }}\left(\bar{r}_{0}^{\prime}-a t\right)\right)$

where $\bar{R}_{0}$ and $\bar{r}_{0}^{\prime}$ are the mean capillary radii before swelling between the individual yarns, and between the elementary fibres, respectively. Constants $b$ and $a$ express the swelling rates of a yarn and of an elementary fibre, respectively.

Inserting Eq. (3) (before swelling: $(c \bar{r})_{i n i}$ corresponding to $\bar{R}_{0}$ and $\bar{r}_{0}$ ) into Eq. (4), the following equation is obtained:

$(c \bar{r})(t)=(c \bar{r})_{i n i}-c t\left(\left(1-\varnothing_{y a r n}\right) b+\varnothing_{\text {yarn }} a\right)$

which expresses for the evolution of the geometric product $c \bar{r}$ during capillary wicking in natural fibres taking into account the fibre swelling at a double scale. In this equation, parameters such as $\varnothing_{\text {yarn }}, b, a, \bar{R}_{0}$, and $\bar{r}_{0}^{\prime}$ can be determined experimentally.

It must be noted that modifications of porosity $\epsilon(t)$ related to the variation of the geometric product $(c \bar{r})(t)$ are taken into account in the 
proposed model the same way as in the previous model [21].

\subsection{Pore radius diminution}

It should be noted that fibres and yarns swell until their maximal dimensions in a definite time. Assuming tortuosity as a constant, the geometric product $c \bar{r}$ thus varies over time until its final lower value $(c \bar{r})_{f i n}$ is reached [21]. Therefore, $(c \bar{r})_{f i n}$ can be defined from minimal mean capillary radius. In the previous model [21], this parameter was limited to a decrease of $30 \%$ because fibres swelled of around this value and also the model could not hold further diminution of mean capillary radius. The present model allows diminution down to $50 \%$ of initial mean capillary radius value, which is the chosen minimal value to extend the time range of validity of the model.

Eq. (7) gives the boundary condition for $c \bar{r}$, taking into account the maximal swelling of fibres:

$$
\begin{aligned}
& \text { if }(c \bar{r})(t)>(c \bar{r})_{f i n} \\
& (c \bar{r})(t)=(c \bar{r})_{i n i}-c t\left(\left(1-\varnothing_{\text {yarn }}\right) b+\varnothing_{\text {yarn }} a\right)
\end{aligned}
$$

else

$(c \bar{r})(t)=(c \bar{r})_{\text {fin }}$

\subsection{Washburn's relation with swelling}

Feeding the proposed modification of porosity, and of pore radius diminution (Section 2.2 and Section 2.3), together with using initial wicking parameter $\left((\boldsymbol{c} \overline{\boldsymbol{r}})_{i n i}\right.$ is determined experimentally) into the Washburn's equation (Eq. (2)), it is noted that the apparent advancing contact angle $\theta_{\boldsymbol{a}}$ and the parameter accounting for tortuosity $c$ in Eq. (2) still need to be identified. These two parameters are obtained when fitting the model to the experimental data of wicking (presented in Section 4.4).

The proposed model implicitly considers an important assumption. Namely, the entire volume of the fibre sample begins to swell when the wicking starts. In other words, no differential or incremental swelling is specified in each position along the elementary fibres and yarns when liquid starts to be in contact with the sample. This assumption is supposed to be valid due to the similar time scales of the fibre swelling and the wicking in the present work. For more complex bio-based preforms, deeper analysis requiring a numerical model will be needed. It will be the main focus of further studies.

\section{Materials and methods}

Experiments were designed to investigate capillary effects coupled with swelling during wicking of water in flax fabrics. Wicking test was first performed at the macro-scale of synthetic and natural fibre fabrics, i.e. carbon fabrics and flax fabrics in the warp direction. Carbon fabrics were used in this test to validate the method at high $V_{f}$. Swelling tests were then carried out at the micro-scale on yarns and elementary fibres for natural fibres only.

\subsection{Materials}

The reinforcements are quasi-unidirectional fabrics of carbon and flax. Carbon fabrics were provided by Hexcel with commercial designation $48580^{\circledR}$. The fabrics contain $3 \%$ of glass yarns in volume, and have areal weight of $540 \mathrm{~g} / \mathrm{m}^{2}$ and density of $1.8 \mathrm{~g} / \mathrm{cm}^{3}$. Flax fabrics were supplied by Libeco with material code FLAXDRY UD $180^{\circledR}$. Their areal weight and density are $180 \mathrm{~g} / \mathrm{m}^{2}$ and $1.45 \mathrm{~g} / \mathrm{cm}^{3}$, respectively.

The cylindrical sample holder in which fibres were placed is made of poly(methyl methacrylate) polymer, it has an inner radius of $6 \mathrm{~mm}$ and a height of $20 \mathrm{~mm}$. The tube was cleaned with sulfochromic acid (50\% of a saturated solution of potassium dichromate and $50 \%$ of
Table 1

Characteristics of test liquids at $20^{\circ} \mathrm{C}$

\begin{tabular}{llllll}
\hline & $\rho\left(\mathrm{g} / \mathrm{cm}^{3}\right)$ & $\eta(\mathrm{mPa} . \mathrm{s})$ & $\gamma_{L}(\mathrm{mN} / \mathrm{m})$ & $\gamma_{L}^{p}(\mathrm{mN} / \mathrm{m})$ & $\gamma_{L}^{d}(\mathrm{mN} / \mathrm{m})$ \\
\hline n-Hexane & 0.659 & 0.32 & 18.4 & 0.0 & 18.4 \\
Water & 0.998 & 1.00 & 72.8 & 51.0 & 21.8
\end{tabular}

With $\rho, \eta, \gamma_{L}, \gamma_{L}^{p}$, and $\gamma_{L}^{d}$ representing respectively the density, viscosity, surface tension and its polar and dispersive components, of liquid which are found in literature [24]

concentrated sulfuric acid), rinsed with water and dried at ambient condition before testing.

$\mathrm{n}$-Hexane and distilled water were selected as reference liquids for this study. n-Hexane is a totally wetting liquid, meaning that the advancing contact angle $\theta_{a}$ is supposed to be zero. This chemical is suitable for determination of initial non-swollen geometric parameter of the fibrous medium $(c \bar{r})_{i n i}$. Distilled water was used to determine the apparent advancing contact angle $\theta_{a}$ from wicking tests, and was also used for swelling tests on elementary flax fibres and yarns. Some characteristics of n-Hexane and distilled water such as surface tension, density, and viscosity are presented in Table 1.

\subsection{Methods}

\subsubsection{Wicking tests}

The details of procedure were well described in previous works $[28,29]$. In this study, the amount of carbon and flax fabrics were determined so as to get a global fibre volume fraction $V_{f}$ of $60 \%$ which is higher than in previous studies $[21,28]$. These fabrics were then rolled along the transverse direction, and inserted into the cylindrical tube. Capillary wicking was performed with a tensiometer (DCAT11, DataPhysics Instrument $\mathrm{GmbH}$, Filderstadt, Germany). The tube was clamped to the tensiometer and the vessel with test liquid moved with a speed of $0.5 \mathrm{~mm} / \mathrm{s}$ up to the detection of the holder. The holder is linked to an electronic microbalance with a resolution of $10 \mu \mathrm{g}$. The data were then recorded with the square of mass gain as a function of time. After measurement with $\mathrm{n}$-Hexane, each sample was dried at $60{ }^{\circ} \mathrm{C}$ for at least $2 \mathrm{~h}$. Their mass loss related to evaporation of n-Hexane was checked with microbalance until the weight of sample did not change before measuring with water. All the wicking tests were performed at $20{ }^{\circ} \mathrm{C}$. For each fabric, at least nine samples were tested.

To determine the initial geometric product $(c \bar{r})_{i n i}$, the procedure is as follows. In constant porous medium, the evolution of square mass gain as a function of time was observed linearly with both $n$-Hexane and water as described in conventional Washburn's equation. An example of linear fits for carbon fabrics is presented in Fig. 2. The geometric product $c \bar{r}$ is determined in Eq. (2) using $n$-Hexane, for which the apparent advancing contact angle $\theta_{a}$ is supposed to be $0^{\circ}$. Because the porous medium remains constants during wicking, the $c \bar{r}$ obtained is considered as $(c \bar{r})_{i n i}$. From linear fits of wicking with water and knowing $(c \bar{r})_{i n i}$, the apparent advancing contact angle with water is then determined.

\subsubsection{Mean capillary radius between yarns by $X$-ray tomography}

In order to evaluate the fabric internal structure in 3D within the entire tube area, nanofocus X-ray computed tomography (nano-CT) was used. Measurements were performed with a Phoenix NanoTom S (GE Measurements and Control solutions, Wunstorf, Gernany). The device was equipped with a tungsten target and the applied voltage and current were $100 \mathrm{kV}$ and $100 \mu \mathrm{A}$, respectively. After scanning, 3D images were reconstructed from the radiographs using "Phoenix datos $\mid$ x 2.5" reconstruction software.

The images obtained by tomography were then analysed by the image analysis software ImageJ to determine the surface ratio of yarns $\varnothing_{\text {yarn }}$ as well as the mean capillary radius between the yarns before 


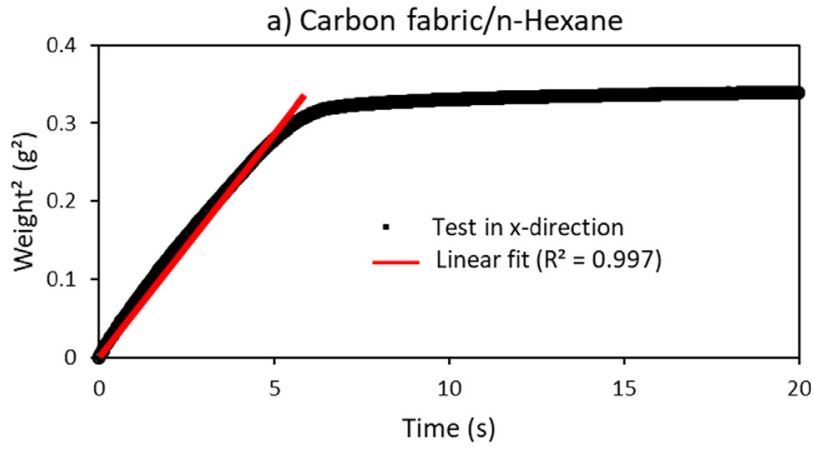

b) Carbon fabric/water

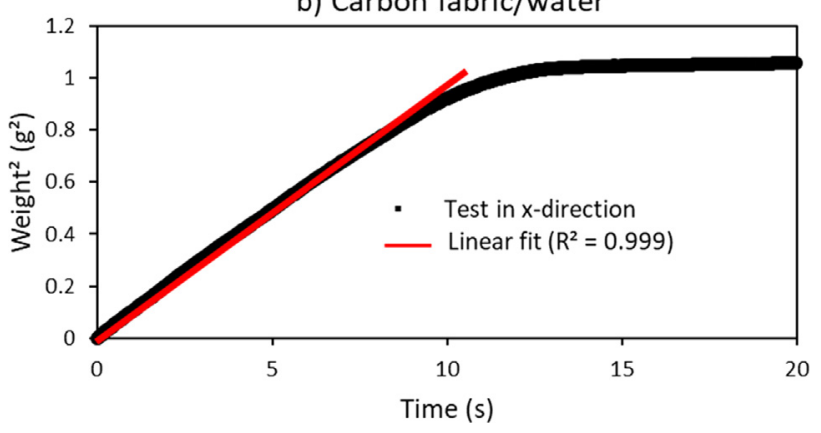

Fig. 2. Linear fit of a wicking test for carbon fabrics at $60 \% \mathrm{~V}_{\mathrm{f}}$ with (a) $n$ Hexane and (b) distilled water. (For interpretation of the references to colour in this figure legend, the reader is referred to the web version of this article.)

swelling $\bar{R}_{0}$. This software was used to determine the pore volume percentage. Flax fabrics were studied in dry condition and saturated with water (Fig. 3).

\subsubsection{Swelling rate and mean capillary radius between elementary fibres by optical microscopy}

In order to determine the swelling rate of flax elementary fibres and individual yarns, an optical microscope (Laborlux 11 POLS, Leitz, Germany) equipped with a "mon-CDD Leica DFC420" digital camera (resolution $1728 \times 1296$ pixels) and an image acquisition software Archimed $^{\circledR}$ (Microvision Instruments, France) has been used. Elementary fibres (extracted from yarns in dry condition) and yarns were positioned in a container made of two glass plates separated with double-sided tape. Distilled water was introduced by capillary forces between the two plates until the water filled the container and fibre swelling phenomenon occurred. The space between the two plates was sufficient for the fibres to freely move in water. No tension was applied to the elementary fibres and yarns. Four to six images per second were recorded from the time of water introduction, until fibres reached a stabilized swollen state. The ImageJ software was used to measure fibre diameter changing over time (assuming a circular section). Because diameter of elementary fibres and yarns vary along their length and greatly change from one to another, diameters were measured at least at five different positions (Figs. 4 and 5). It should be pointed out that in the case of flax yarns, diameter measurements were made more difficult due to fibrillation as seen in Fig. 5. At least five samples of elementary fibres and yarns were analyzed for the swelling test.

Optical microscope was also chosen to determine the mean capillary radius between elementary fibres before swelling $\dot{r}_{0}$. It would be very complicated to measure the $\bar{r}_{0}^{\prime}$ with tomography since the minimal voxel size (around $800 \mu \mathrm{m}^{3}$ ) obtained in a single yarn is much higher than the diameter of elementary fibres and spaces in between them. Therefore, images taken from an epoxy based composite reinforced by flax quasiUD fabrics (Fig. 1) were analyzed with ImageJ in order to estimate $\bar{r}_{0}$. Those samples were manufactured in dry conditions under vacuum, assuming that the resin had no significant swelling effect on fibres. It is also assumed that the $\bar{r}_{0}^{\prime}$ does not change much with the $V_{f}$.

\section{Results and discussion}

\subsection{Validation from wicking in carbon fabrics}

To validate that this method can be applied for reinforcements with $V_{f}$ of $60 \%$, the wicking tests were carried out with n-Hexane on carbon fabrics, following the procedure detailed in Section 3.2.1. The evolution of square of mass gain as a function of time was monitored, as presented in Fig. 6a. The linearity confirms the validity of Washburn's equation with the totally wetting liquid for carbon fabrics. As expected, the equilibrium mass gain in this study at $60 \% V_{f}$ is lower than the one in a previous study at $40 \% V_{f}$ [28]. This is logical since the mass of liquid inside the sample holder decreases with the void space available. Table 2 presents the results of linear fitting with the correlation coefficient $\left(R^{2}\right)$, and subsequently the determined initial geometric product $(c \bar{r})_{i n i}$ for each test. The mean result of geometric product $(c \bar{r})_{i n i}$ was then calculated as $2.34 \pm 0.35 \mu \mathrm{m}$. This mean value is lower than the one calculated at $40 \% V_{f}(12.1 \pm 1.5 \mu \mathrm{m})$ [28]. This is also expected since the $\bar{r}$ decreases as $V_{f}$ increases [21].

Similar tests were also performed with distilled water to determine an apparent advancing contact angle $\theta_{a}$. The results of capillary wicking with water are presented in Fig. 6b. The linear slope is also observed, and hence the Washburn's equation can be applied. The advancing contact angle is calculated based on both the slope of the linear fit and the geometric product determined with $n$-Hexane. The result of each test is presented in Table 2, and the mean calculated value of the advancing contact angle was $55.4^{\circ} \pm 3.8^{\circ}$. The linearity of wicking and the resulting parameters validate that this method can be used for $V_{f}$ up to $60 \%$.
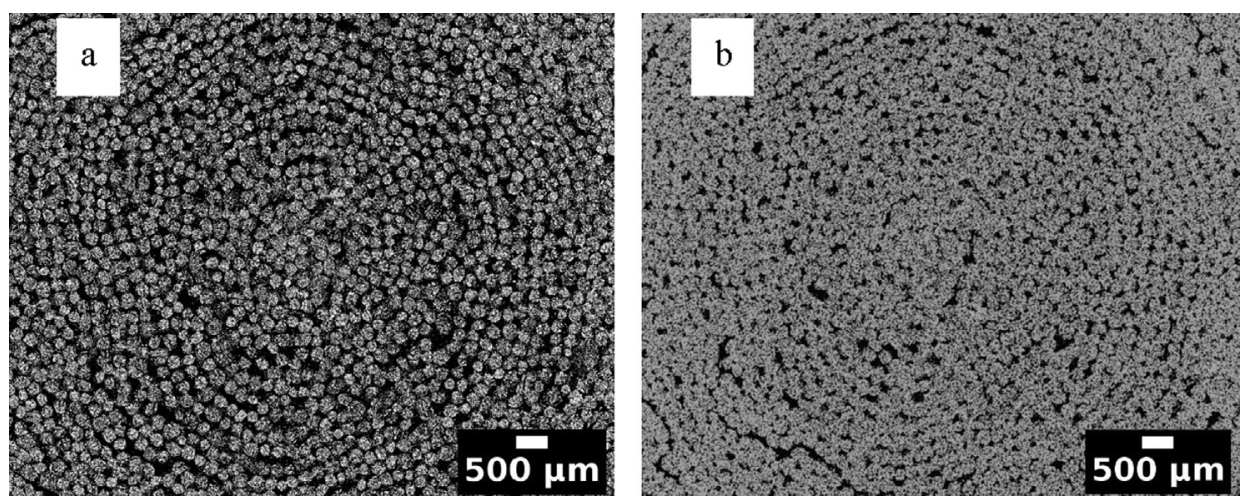

Fig. 3. Nano-CT cross-sectional images of dry flax yarns within the cylindrical tube with initial $60 \% \mathrm{~V}_{\mathrm{f}}$ before (a) and after (b) swelling in water (only one sample for each material and condition was scanned by nano-CT due to complexity of the set-up). 

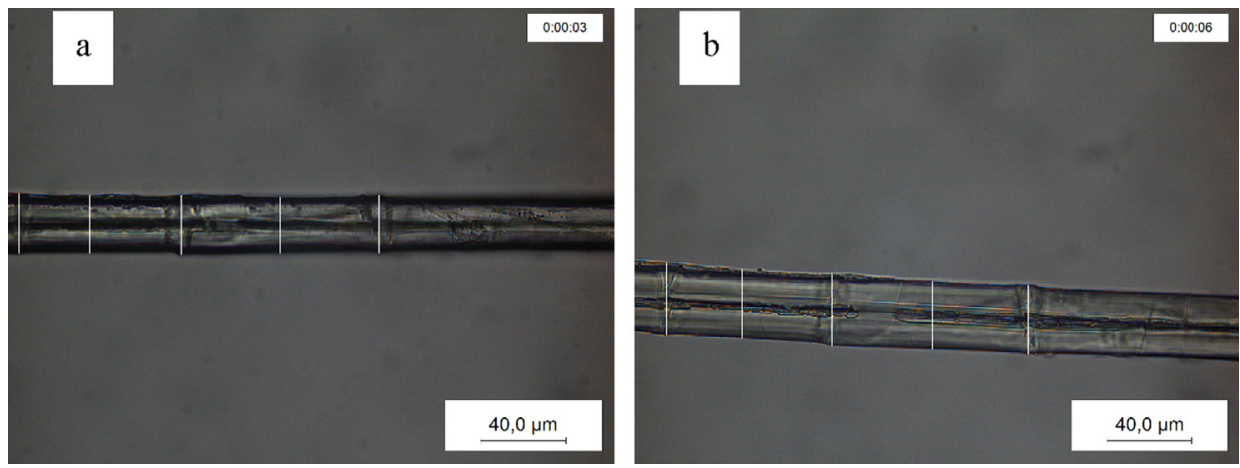

Fig. 4. Optical measurements of diameters for an elementary flax fibre before (a) and after (b) swelling in distilled water. The white lines marked on the fibres represent the positions where fibre diameters were measured. Caution was paid to measure the diameters at the same position along the fibre axis before and after swelling.
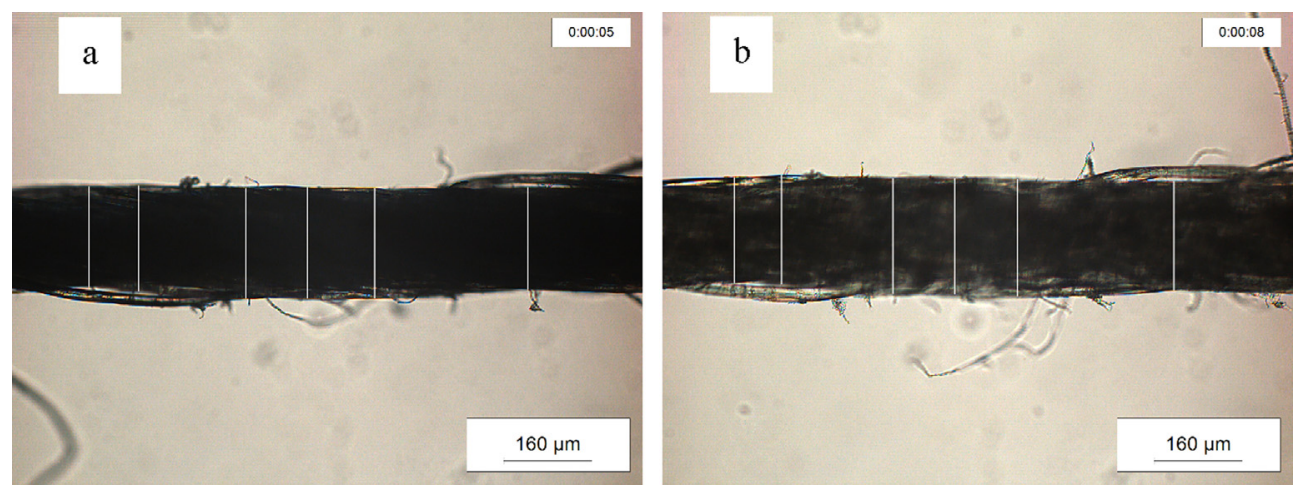

Fig. 5. Optical measurements of diameters for a flax yarn before (a) and after (b) swelling in distilled water. The white lines marked on the yarns represent the positions where yarns diameters were measured. Caution was paid to measure the diameters at the same position along the yarn axis before and after swelling.
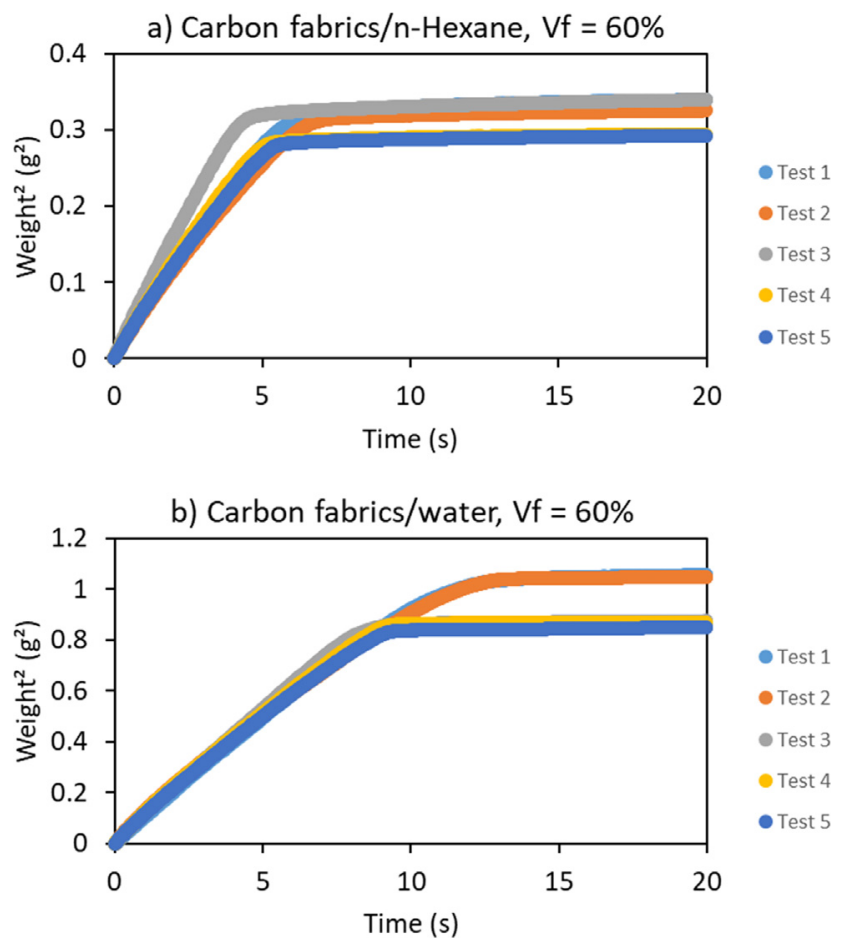

Fig. 6. Wicking curves in warp direction of carbon fabrics with (a) $n$-Hexane and (b) distilled water at $60 \% \mathrm{~V}_{\mathrm{f}}$. (For interpretation of the references to colour in this figure legend, the reader is referred to the web version of this article.)

\subsection{Wicking in flax fabrics}

Using the same method as for carbon fabrics, the initial geometric product $(c \bar{r})_{i n i}$ was determined with capillary wicking of flax fibres with
Table 2

Results of wicking tests for carbon fabrics with $n$-Hexane and water at $60 \% \mathrm{~V}_{\mathrm{f}}$.

\begin{tabular}{lllllll}
\hline Carbon & \multicolumn{7}{l}{$n$-Hexane } & \multicolumn{5}{c}{ Water } \\
\cline { 2 - 7 } & Linear slope & $R^{2}$ & $(c \bar{r})_{\text {ini }}(\mu \mathrm{m})$ & Linear slope & $R^{2}$ & $\theta_{a}\left({ }^{\circ}\right)$ \\
\hline Sample 1 & 0.057 & 0.9973 & 2.24 & 0.097 & 0.9996 & 54.2 \\
Sample 2 & 0.052 & 0.9974 & 2.05 & 0.093 & 0.9968 & 52.0 \\
Sample 3 & 0.075 & 0.9988 & 2.95 & 0.103 & 0.9997 & 61.8 \\
Sample 4 & 0.059 & 0.9985 & 2.30 & 0.097 & 0.9984 & 55.2 \\
Sample 5 & 0.055 & 0.9977 & 2.15 & 0.095 & 0.9987 & 53.5 \\
\hline
\end{tabular}

n-Hexane. Fig. 7a shows the results of wicking curves for five different samples. The linearity was also observed, supporting that Washburn's equation can be used. The results of wicking tests for flax fabrics are presented in Table 3 and a mean $(c \bar{r})_{i n i}$ of $3.77 \pm 0.37 \mu \mathrm{m}$ was obtained.

Regarding capillary wicking with water, non-linear trends were observed with a large variation between the samples (Fig. 7b). Consequently, Washburn's equation cannot be applied. As pointed out in the introduction, the chemical composition and surface properties of natural fibres and the variations of diameter along their length lead to heterogeneous wetting as well as complex tortuosity of pore between the fibres (in both individual yarns and elementary fibres), influencing the capillary wicking behaviour $[21,28,30]$. Furthermore, the swelling of flax fibres during wicking causes an increase of fibre diameter within the cylindrical tube, and hence a decrease of porosity, and of the mean capillary radius $\bar{r}$. As a result, capillary rise speed evolves during the wicking test (i.e. it is decreased), and the equilibrium weight is more slowly reached. It should be pointed out that the equilibrium weight is also determined by water sorption within the flax fibre cell walls which occurs concomitantly with fibre swelling. 

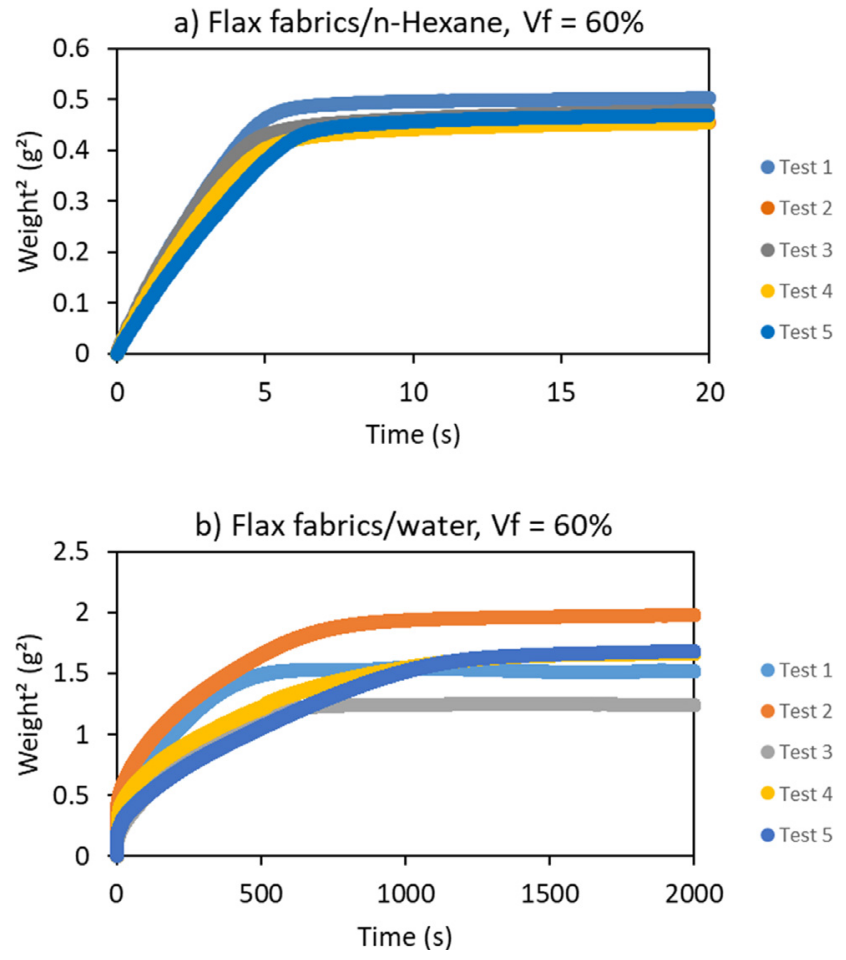

Fig. 7. Wicking curves in warp direction of flax fabrics with (a) $n$-Hexane and (b) distilled water at $60 \% \mathrm{~V}_{\mathrm{f}}$. (For interpretation of the references to colour in this figure legend, the reader is referred to the web version of this article.)

Table 3

Results of wicking tests for flax fabrics with n-Hexane at $60 \% \mathrm{~V}_{\mathrm{f}}$.

\begin{tabular}{llll}
\hline Flax & Linear slope & $R^{2}$ & $(c \bar{r})_{\text {ini }}(\mu \mathrm{m})$ \\
\hline Sample 1 & 0.106 & 0.9963 & 4.14 \\
Sample 2 & 0.093 & 0.9953 & 3.64 \\
Sample 3 & 0.104 & 0.9957 & 4.09 \\
Sample 4 & 0.095 & 0.9953 & 3.73 \\
Sample 5 & 0.082 & 0.9972 & 3.23 \\
\hline
\end{tabular}

\subsection{Measurement of capillary radii, surface ratio of yarn, and swelling}

Before swelling, $\bar{R}_{0}$ and $\bar{r}_{0}^{\prime}$ are considered as the largest mean capillary radii between individual yarns and between elementary fibres, respectively. $\varnothing_{\text {yarn }}$ is defined as the surface ratio of yarns at the scale of composites, taking into account the largest mean radius between individual yarns $\bar{R}_{0}$ and the largest mean capillary radius between elementary fibres $\bar{r}_{0}^{\prime}$. Their values (presented in Table 4) were determined by images taken by X-ray tomography (for $\bar{R}_{0}$ and $\varnothing_{\text {yarn }}$ ) and optical

Table 4

Results of measurement related to swelling parameters of flax fibres.

\begin{tabular}{lllll}
\hline $\bar{R}_{0}(\mu \mathrm{m})$ & $\dot{\bar{r}}_{0}^{\prime}(\mu \mathrm{m})$ & $\varnothing_{\text {yarn }}$ & $b(\mu \mathrm{m} / \mathrm{s})$ & $a(\mu \mathrm{m} / \mathrm{s})$ \\
\hline $108.84 \pm 27.31$ & $6.63 \pm 2.55$ & $0.66^{*}$ & $4.76 \pm 2.66$ & $2.11 \pm 0.66$
\end{tabular}

With $\bar{R}_{0}, \bar{r}_{0}, \varnothing_{\text {yarn }}, b$ and $a$ representing respectively the mean capillary radius before swelling between yarns, the mean capillary radius before swelling between elementary fibres, the surface ratio of yarns at the scale of composites, the swelling rate of a yarn, and the swelling rate of an elementary fibre.

* Only one sample was scanned by nano-CT due to complexity of the set-up, hence no standard deviation is given. It must be noted that $\varnothing_{\text {yarn }}$ (as defined in Section 2.2) only considers a surface ratio of yarns on an overall section of sample, and it thus does not consider spaces in between elementary fibres. Therefore, the value of $\varnothing_{\text {yarn }}$ obtained does not correspond to $60 \% \mathrm{~V}_{\mathrm{f}}$ and is thus necessary higher.
Table 5

Diameter distribution of an elementary flax fibre (Fig. 4a) and of a flax yarn (Fig. 5a) before wetting.

\begin{tabular}{lllllll}
\hline Fibres & $\begin{array}{l}\text { Position } \\
(\mu \mathrm{m})\end{array}$ & $\begin{array}{l}\text { Position } \\
2(\mu \mathrm{m})\end{array}$ & $\begin{array}{l}\text { Position } \\
3(\mu \mathrm{m})\end{array}$ & $\begin{array}{l}\text { Position } \\
4(\mu \mathrm{m})\end{array}$ & $\begin{array}{l}\text { Position } \\
5(\mu \mathrm{m})\end{array}$ & $\begin{array}{l}\text { Position } \\
6(\mu \mathrm{m})\end{array}$ \\
\hline Elementary & 28.40 & 27.24 & 27.98 & 26.09 & 27.12 & - \\
Yarn & 181.93 & 188.21 & 200.33 & 201.65 & 200.33 & 185.62 \\
\hline
\end{tabular}
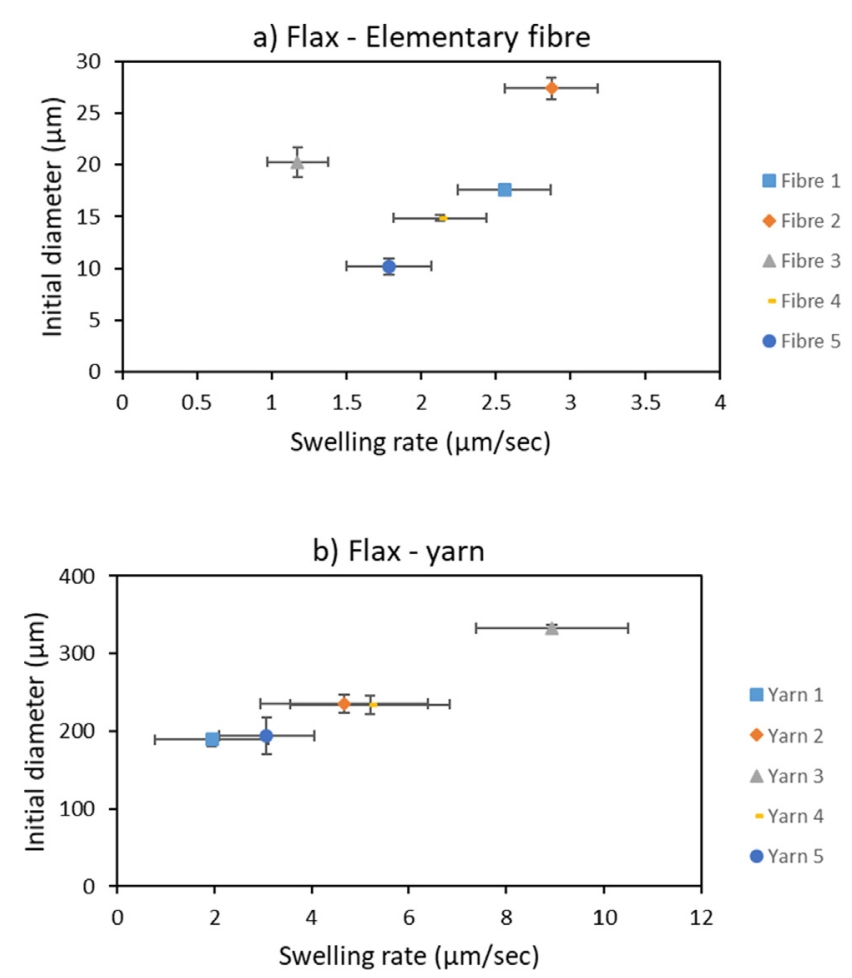

Fig. 8. Swelling rate of (a) elementary fibre and (b) yarn versus initial diameter. (For interpretation of the references to colour in this figure legend, the reader is referred to the web version of this article.)

microscopy (for $\bar{r}_{0}^{\prime}$ ) and then image analysed with ImageJ as described in Section 3.2.2 and Section 3.2.3.

As mentioned earlier, the diameter of an elementary flax fibre as well as the one of a yarn are not uniform. Table 5 shows results of diameter distribution before wetting. This is in line with several studies which show that flax fibres exhibit a large scatter of diameter along their length $[16,21,31,32]$. After wetting in water, elementary flax fibres and yarns have significantly swelled as clearly seen in Figs. 4 and 5. Fig. 8 presents the results of swelling tests with water for elementary fibres and yarns. It is noticed that faster swelling rates were related to larger initial diameters for both elementary fibres and yarns. The mean values of their swelling rate are given in Table 4, respectively coefficients $b$ and $a$ in Eq. (4).

\subsection{Comparison between experiment and model}

Capillary wicking is well described by conventional Washburn's equation for invariant porous structure morphology. However, wicking curves presented in Fig. $7 \mathrm{~b}$ evidence that changes in the porous medium during wicking in case of natural fibres require modification of the Washburn's equation. Therefore, the modified Washburn's model proposed in Section 2.2 was applied to fit experimental data.

As mentioned in Section 2.4, the apparent advancing contact angle $\theta_{a}$ and the parameter accounting for tortuosity $c$ in Eq. (2) require to be identified to fit the experimental data. As observed in Fig. 9a, based on the modified Washburn's equation, the values of $\theta_{a}$ and $c$ could be 


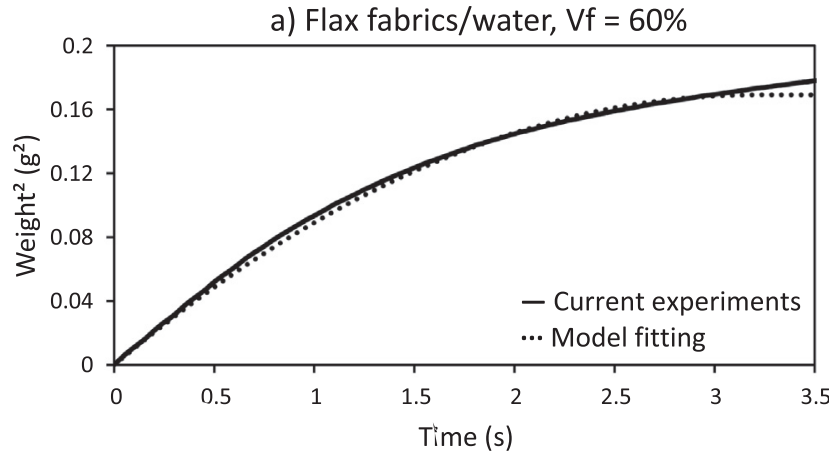

b) Flax fabrics/water, $\mathrm{Vf}=30,35,40 \%$

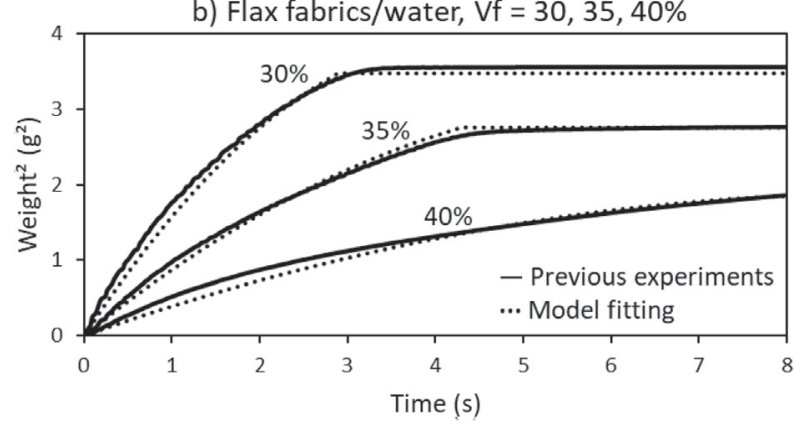

Fig. 9. Fitting of experimental wicking data with the modified Washburn's equation for flax fabrics with water at (a) $60 \% \mathrm{~V}_{\mathrm{f}}$ and (b) $30,35,40 \% \mathrm{~V}_{\mathrm{f}}$ [17].

determined, and the model satisfactory fits the experimental data with values of $\theta_{a}$ and $c$ of $67.5^{\circ}$ and 0.166 , respectively. Another originality of the proposed model compared to the previous one is that it allows to determine the value of the factor $c$ related to tortuosity that becomes an independent and adjustable parameter from the geometric product $c \bar{r}$. This is the first time that $c$ and $\bar{r}$ are considered separately.

Moreover, the modified Washburn's model holds well at the first three seconds of wicking. The wicking of water in natural fibre fabrics at $60 \% V_{f}$ is more complex than at lower $V_{f}$. This is in agreement with the previous work [21] which showed that the effect of swelling is more significant at higher fibre volume fraction. This is also why the previous model [21] cannot describe phenomenon occurring at $V_{f}$ higher than $40 \%$. In addition, at $40 \%$, the previous model could represent experimental data for four seconds whereas the proposed model holds for eight seconds as seen in Fig. 9b (twice longer). The present model can also satisfactory predict experimental data at $V_{f}$ of $30 \%$ and $35 \%$ [21], which highlights its versatility. Even if the previous model was predicting the wicking at low $V_{f}(30-35 \%)$, it was not able to cover the full $V_{f}$ range (30-60\%). The present one has shown its reliability from $30 \%$ to $60 \%$ of $V_{f}$. Therefore, the proposed modified Washburn's model that considers a double scale of swelling (elementary fibres and yarns) and setting measured swelling rates, expands the range of validity of the previous model [21] in terms of $V_{f}$, and also improves it for lower $V_{f}$.

\section{Conclusions}

The understanding and prediction of capillary wicking in natural fibre fabrics is determinant for improving manufacturing with LCM processes and the quality of composite parts. An experimental methodology was developed to evaluate capillary rise, taking into account the multi-scale swelling effects in natural fibres. In the present work, swelling was studied at two scales: elementary fibres and individual yarns. Wicking tests were performed on quasi-unidirectional carbon and flax fabrics at $60 \% V_{f}$. Carbon fabrics showed a linear trend following the Washburn's equation, while a non-linear trend was observed with flax fabrics for wicking tests in water. This highlights the need to take into account swelling effects in natural fibres to better describe capillary wicking. Based on a modified Washburn's equation that considers a double scale of swelling and experimental swelling rates, the capillary parameters have been determined experimentally for both reinforcements. The proposed model has shown to fit very accurately experimental data during the first three seconds of wicking, at $V_{f}$ up to $60 \%$. At lower $V_{f}$, i.e. $40 \%$, the proposed model can fit experimental data for eight seconds which shows the improvement to twice longer duration compared to the standard model [21]. A numerical implementation of this model would allow taking into account the differential swelling of fibres depending on the duration that they were immersed in liquids. Particularly, elementary fibres and yarns at the bottom of the sample start swelling before the ones at the middle. This effect, which would be addressed in further works, is not included in the proposed model and would extend drastically its domain of validity.

\section{CRediT authorship contribution statement}

H.N. Vo: Writing - original draft, Methodology, Investigation, Formal analysis. M.F. Pucci: Conceptualization, Validation, Investigation, Writing - review \& editing. S. Corn: Conceptualization, Methodology. N. Le Moigne: Conceptualization, Methodology. W. Garat: Methodology. S. Drapier: Writing - review \& editing. P.J. Liotier: Conceptualization, Project administration, Supervision, Formal analysis, Writing - review \& editing.

\section{Declaration of Competing Interest}

The authors declare that they have no known competing financial interests or personal relationships that could have appeared to influence the work reported in this paper.

\section{Acknowledgments}

The authors would like to thank O. Valfort for performing the computed tomography machine of reinforcement materials.

\section{References}

[1] Baley C. Analysis of the flax fibres tensile behaviour and analysis of the tensile stiffness increase. Compos - Part A Appl Sci Manuf 2002;33:939-48. https://doi. org/10.1016/S1359-835X(02)00040-4.

[2] Le Duigou A, Davies P, Baley C. Environmental impact analysis of the production of flax fibres to be used as composite material reinforcement. J Biobased Mater Bioenergy 2011;5:153-65. https://doi.org/10.1166/jbmb.2011.1116.

[3] Tran LQN, Fuentes CA, Dupont-Gillain C, Van Vuure AW, Verpoest I. Wetting analysis and surface characterisation of coir fibres used as reinforcement for composites. Colloids Surfaces A Physicochem Eng Asp 2011;377:251-60. https://doi. org/10.1016/j.colsurfa.2011.01.023.

[4] Fuentes CA, Tran LQN, Dupont-Gillain C, Vanderlinden W, De Feyter S, Van Vuure AW, et al. Wetting behaviour and surface properties of technical bamboo fibres. Colloids Surfaces A Physicochem Eng Asp 2011;380:89-99. https://doi.org/10. 1016/j.colsurfa.2011.02.032.

[5] Placet V, Trivaudey F, Cisse O, Gucheret-Retel V, Boubakar ML. Diameter dependence of the apparent tensile modulus of hemp fibres: A morphological, structural or ultrastructural effect? Compos Part A Appl Sci Manuf 2012;43:275-87. https:// doi.org/10.1016/j.compositesa.2011.10.019.

[6] Wambua P, Ivens J, Verpoest I. Natural fibres: can they replace glass in fibre reinforced plastics? Compos Sci Technol 2003;63:1259-64. https://doi.org/10.1016/ S0266-3538(03)00096-4.

[7] Park CH, Lebel A, Saouab A, Bréard J, Lee Il W. Modeling and simulation of voids and saturation in liquid composite molding processes. Compos Part A Appl Sci Manuf 2011;42:658-68. https://doi.org/10.1016/j.compositesa.2011.02.005.

[8] Salvatori D, Caglar B, Michaud V. 3D spacers enhance flow kinetics in resin transfer molding with woven fabrics. Compos Part A Appl Sci Manuf 2019;119:206-16. https://doi.org/10.1016/j.compositesa.2019.01.023.

[9] Caglar B, Tekin C, Karasu F, Michaud V. Assessment of capillary phenomena in liquid composite molding. Compos Part A Appl Sci Manuf 2019;120:73-83. https:// doi.org/10.1016/j.compositesa.2019.02.018.

[10] Umer R, Bickerton S, Fernyhough A. The effect of yarn length and diameter on permeability and compaction response of flax fibre mats. Compos Part A Appl Sci Manuf 2011;42:723-32. https://doi.org/10.1016/j.compositesa.2011.02.010.

[11] Francucci G, Rodriguez E. Processing of plant fiber composites by liquid molding 
techniques: An overview. Polym Compos 2016;37:718-33. https://doi.org/10. 1002/pc.23229.

[12] Charlet K, Baley C, Morvan C, Jernot JP, Gomina M, Bréard J. Characteristics of Hermès flax fibres as a function of their location in the stem and properties of the derived unidirectional composites. Compos Part A Appl Sci Manuf 2007;38:1912-21. https://doi.org/10.1016/j.compositesa.2007.03.006.

[13] Schuchardtl DR, Berg JC. Liquid transport in composite cellulose -superabsorbent fiber networks. Wood Fiber Sci 1991;23:342-57.

[14] Masoodi R, Pillai KM. Darcy's law-based model for wicking in paper-like swelling porous media. AIChE J 2010;56:2257-67. https://doi.org/10.1002/aic.12163.

[15] Washburn EW. Note on a method of determining the distribution of pore sizes in a porous material. Proc Natl Acad Sci USA 1921:115-6. https://doi.org/10.1103/ PhysRev.17.273.

[16] Nguyen VH, Lagardère M, Park CH, Panier S. Permeability of natural fiber reinforcement for liquid composite molding processes. J Mater Sci 2014;49:6449-58. https://doi.org/10.1007/s10853-014-8374-1.

[17] Nguyen VH, Deléglise-Lagardère M, Park CH. Modeling of resin flow in natural fiber reinforcement for liquid composite molding processes. Compos Sci Technol 2015;113:38-45. https://doi.org/10.1016/j.compscitech.2015.03.016.

[18] Charlet K, Jernot JP, Breard J, Gomina M. Scattering of morphological and mechanical properties of flax fibres. Ind Crops Prod 2010;32:220-4. https://doi.org/ 10.1016/j.indcrop.2010.04.015.

[19] Pucci MF, Duchemin B, Gomina M, Bréard J. Temperature effect on dynamic wetting of cellulosic substrates by molten polymers for composite processing. Compos Part A Appl Sci Manuf 2018;114:307-15. https://doi.org/10.1016/j.compositesa. 2018.08.031.

[20] Masoodi R, Pillai KM. A study on moisture absorption and swelling in bio-based jute-epoxy composites. J Reinf Plast Compos 2012;31:285-94. https://doi.org/10. 1177/0731684411434654.

[21] Pucci MF, Liotier PJ, Drapier S. Capillary wicking in flax fabrics - Effects of swelling in water. Colloids Surfaces A Physicochem Eng Asp 2016;498:176-84. https://doi. org/10.1016/j.colsurfa.2016.03.050.

[22] Pucci MF, Liotier PJ, Seveno D, Fuentes C, Van Vuure A, Drapier S. Wetting and swelling property modifications of elementary flax fibres and their effects on the
Liquid Composite Molding process. Compos Part A Appl Sci Manuf 2017;97:31-40. https://doi.org/10.1016/j.compositesa.2017.02.028.

[23] Joffre T, Isaksson P, Dumont PJJ, du Roscoat SR, Sticko S, Orgéas L, et al. A Method to measure moisture induced swelling properties of a single wood cell. Exp Mech 2016;56:723-33. https://doi.org/10.1007/s11340-015-0119-9.

[24] Garat W, Le Moigne N, Corn S, Beaugrand J, Bergeret A. Swelling of natural fibre bundles under hygro- and hydrothermal conditions: Determination of hydric expansion coefficients by automated laser scanning. Compos Part A Appl Sci Manuf 2020;131:105803https://doi.org/10.1016/j.compositesa.2020.105803.

[25] Francucci G, Rodríguez ES, Vázquez A. Study of saturated and unsaturated permeability in natural fiber fabrics. Compos Part A Appl Sci Manuf 2010;41:16-21. https://doi.org/10.1016/j.compositesa.2009.07.012.

[26] Lee DH, Lee II W, Kang MK. Analysis and minimization of void formation during resin transfer molding process. Compos Sci Technol 2006;66:3281-9. https://doi. org/10.1016/j.compscitech.2005.07.008.

[27] Stuart T, McCall RD, Sharma HSS, Lyons G. Modelling of wicking and moisture interactions of flax and viscose fibres. Carbohydr Polym 2015;123:359-68. https:// doi.org/10.1016/j.carbpol.2015.01.053.

[28] Pucci MF, Liotier PJ, Drapier S. Capillary wicking in a fibrous reinforcement Orthotropic issues to determine the capillary pressure components. Compos Part A Appl Sci Manuf 2015;77:133-41. https://doi.org/10.1016/j.compositesa.2015.05. 031.

[29] Pucci MF, Liotier PJ, Drapier S. Capillary effects on flax fibers - Modification and characterization of the wetting dynamics. Compos Part A Appl Sci Manuf 2015;77:257-65. https://doi.org/10.1016/j.compositesa.2015.03.010.

[30] Pejic BM, Kostic MM, Skundric PD, Praskalo JZ. The effects of hemicelluloses and lignin removal on water uptake behavior of hemp fibers. Bioresour Technol 2008;99:7152-9. https://doi.org/10.1016/j.biortech.2007.12.073.

[31] Baley C, Bourmaud A. Average tensile properties of French elementary flax fibers Mater Lett 2014;122:159-61. https://doi.org/10.1016/j.matlet.2014.02.030.

[32] Garat W, Corn S, Le Moigne N, Beaugrand J, Bergeret A. Analysis of the morphometric variations in natural fibres by automated laser scanning: Towards an efficient and reliable assessment of the cross-sectional area. Compos Part A Appl Sci Manuf 2018;108:114-23. https://doi.org/10.1016/j.compositesa.2018.02.018. 